\title{
Information and Communication Technology in the Fight Against the COVID-19 Pandemic/Infodemic
}

\author{
Authors: \\ Shinji Kobayashi \\ Centre of Public Health, National Institute of Public Health, Saitama, Japan \\ *Correspondence to kobayashi.s.nj@niph.go.jp \\ Disclosure: \\ The author has declared no conflicts of interest. \\ Received: \\ 31.08 .20 \\ Accepted: \\ 17.11 .20 \\ Keywords: \\ Coronavirus disease (COVID-19), eHealth, information and communication technology \\ (ICT), technology.
}

Citation:

EMJ Innov. 2021;5[1]:42-45.

\section{INTRODUCTION}

A new coronavirus infection named coronavirus disease (COVID-19) was discovered in Wuhan, China at the end of 2019, and has rapidly progressed. The World Health Organization (WHO) declared COVID-19 a pandemic in March 2020. COVID-19 was characterised by the rapidity of the outbreak that was accelerated by transportation networks worldwide. Researchers have attempted various approaches to manage COVID-19, such as genome analyses, diagnostic methods, treatments, and prevention. An 'infodemic' situation has developed, whereby misinformation has caused logistical disruptions and resulted in health hazards and shortages of supplies. In Japan, face masks became difficult to find and toilet paper temporarily disappeared from stores because it was thought that it was made from the same paper as masks. ${ }^{1}$ Similar panic buying and stockpiling has occurred worldwide, disrupting logistics. In Iran, a false rumour circulated that methanol was effective against COVID-19, which led to nearly 500 deaths. ${ }^{2}$ In the USA, the President Donald Trump made comments about injecting disinfectant, leading to several people drinking the disinfectant and causing them health problems. ${ }^{3}$ The International Telecommunication Union (ITU) released a statement to make it known that there is no scientific basis for the claim that $5 \mathrm{G}$ network accelerated the COVID-19 pandemic. $^{4}$ Social unrest has increased the stigma attached to those of Asian ethnicity, people who have recovered from COVID-19, and health care workers. ${ }^{5}$ Even before the COVID-19 outbreak, smartphone apps were being utilised to combat infectious diseases, and more apps have since been developed to tackle COVID-19. Though information and communication technology (ICT) have the capacity to cause an infodemic, ICT has been widely applied as a tool against the COVID-19 crisis. The Taiwanese government disclosed that their advanced information technology capacity helped them to achieve far greater control of COVID-19 than other countries. ${ }^{6}$ In this article, the author has reviewed the features of successful ICT approaches against COVID-19 and discussed their effects.

\section{INFORMATION AND COMMUNICATION TECHNOLOGY APPLICATIONS FOR COVID-19}

The ICT applications for the COVID-19 pandemic/ infodemic have been categorised into patient registry, clinical decision support, telemedicine, contact tracing, and digital quarantine. Each category is outlined below (Figure 1). 


\section{Patient Registry/Geographical Information System}

The tracking of patients and their contacts is imperative to public health in the fight against infectious diseases. Johns Hopkins University Department of Public Health, Baltimore, Maryland, USA, developed an interactive geographical information service (GIS) that was developed using ArcGIS (Esri, Redlands, California, USA) to display the number of confirmed cases of and deaths caused by COVID-19 on a map. ${ }^{7}$ This GIS publishes epidemiological data worldwide, as well as the country and regional trends in patient numbers. Comprehensive information is available on maps and graphs (Figure 1). The World Health Organization (WHO) also built a similar website $^{8}$ and, in Japan, the Tokyo Metropolitan Government commissioned a website to visualise data related to COVID-19 (confirmed patients and related deaths). ${ }^{9}$

\section{Clinical Decision Support}

It has also been reported that machine learning of medical images, such as chest radiographs and
CT scans, can be used to accurately diagnose COVID-19-associated pneumonia. ${ }^{10}$ Much of the available data related to COVID-19 are open access, and machine learning models to support clinical decision making have made use of such data. The creation of electronic clinical guidelines has also been reported, whereby implemented data sets have aided diagnosis and treatment of COVID-19."

\section{Telemedicine}

The use of telemeeting and teleworking systems using ICT became popular because of the many regional lockdowns that acted as a countermeasure to the pandemic. In Japan, telemedicine had not previously been adopted widely but now many healthcare providers utilise it for outpatient management and the initial treatment of patients with COVID-19. Various medical conferences have been held as teleconferences using video calling technologies, which has contributed to the spread of the latest medical information, including the latest developments in COVID-19 research.

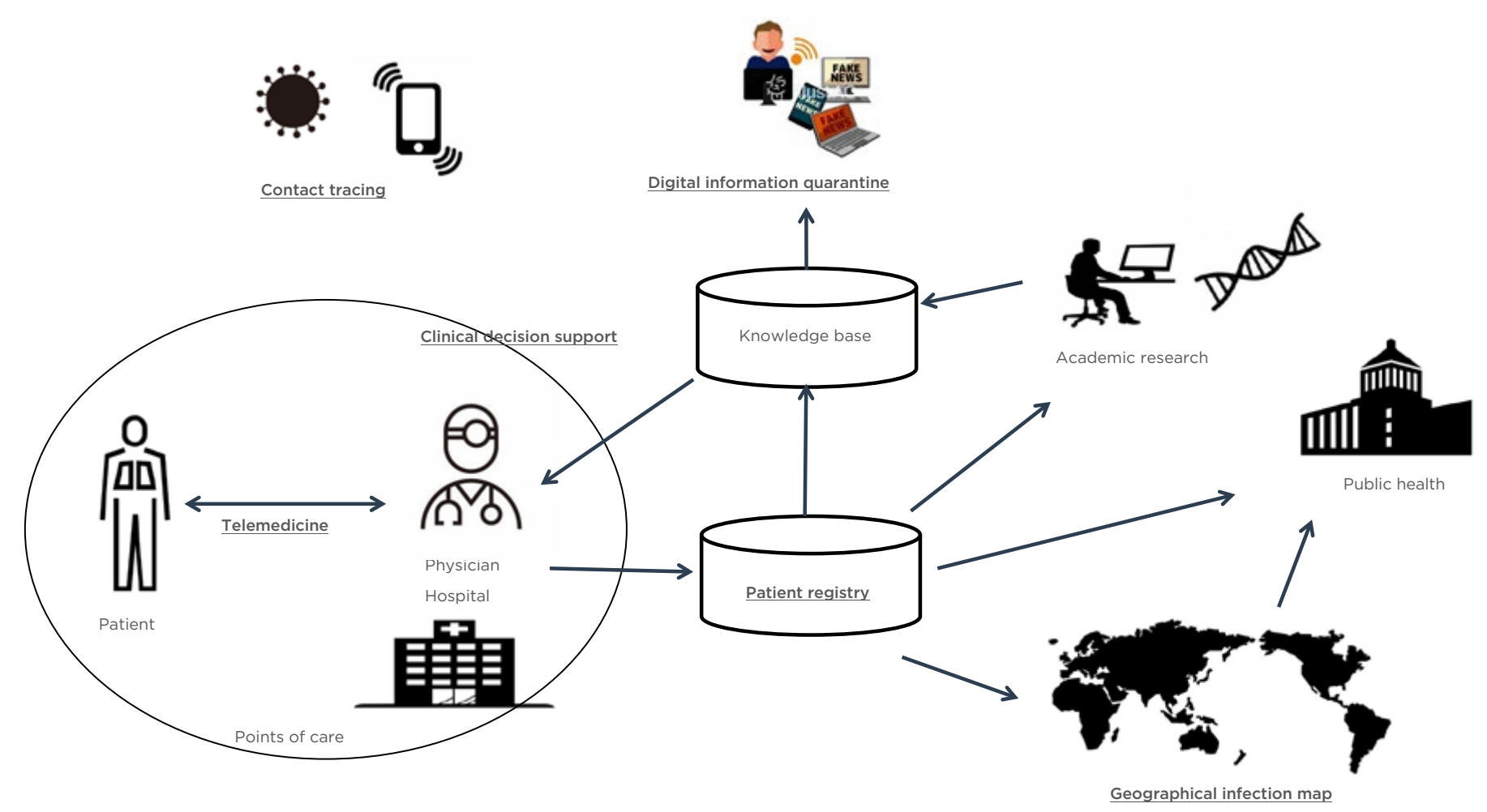

Figure 1: Information and communication technology tools for the coronavirus disease (COVID-19) pandemic/infodemic. 


\section{Contact Tracing}

Digital contact tracing was suggested as a means to help control COVID-19 transmission following both real-world experiences in Singapore and mathematical models. ${ }^{12}$ Both Apple and Google provide a contact tracing application programming interface (API) on their iOS (Apple, Cupertino, California, USA) and Android (Google, Mountain View, California, USA) products. Both companies limited access to the representative agency in the user's country to protect user privacy. Japan and Germany have also developed contact tracing software using Google/Apple API and have shared it with the public as an open-source software.

\section{Digital Quarantine Against Misinformation}

To counter COVID-19-related misinformation, the $\mathrm{WHO}$ and other health organisations of many countries have ensured that accurate information is published. They have also blocked misinformation shared on social media and guided public information. Social media platforms also banned many accounts that propagated conspiracies related to COVID-19.

\section{DISCUSSION}

There are many advantages and disadvantages of utilising ICT in a pandemic, the disadvantages mainly concerning privacy issues and ICT investment. Various ICT tools are being used to fight the current COVID-19 pandemic. The data related to COVID-19 are available as open data, and volunteers have been using it to help control the spread of disease. It is helpful to understand the current epidemic status using websites that visualise the 'heatmap' of patient numbers and graphs (Figure 1). Trials using data for clinical support are underway, and there are high expectations regarding the potential of ICT tools to fight COVID-19.

In contrast, the privacy of infected patients has the capacity to be violated by a breach of the ICT software. Contact tracing could divulge private information, such as the whereabouts of a user and their relationships, to an unintended audience. There is also scepticism about its effectiveness in tracking infection; for example, a contact tracing app was released in Norway but was discontinued because of the low numbers of people infected and the suspicions about privacy implications. The $\mathrm{WHO}$ published a statement in 2016 about ethical considerations during the epidemics of infectious diseases, aiming to avoid the stigma directed at patients by protecting personal privacy. ${ }^{13}$ Germany and Japan are developing their contact tracing app as opensource software in order to improve transparency and manage private information.

Nevertheless, ICT has been widely adopted to fight COVID-19, despite the suspicions the public may have about the technology. Misinformation has generated panic in relation to disasters throughout human history, long before the ICT era. ICT has accelerated the speed of information and misinformation spread and caused an infodemic, a new type of information panic. However, ICT has also delivered authentic information about COVID-19 and become a modern weapon in the fight against the pandemic. Telemedicine has already become part of the healthcare infrastructure to combat COVID-19, and will be the 'new normal' when the pandemic is over. More ICT tools are needed to keep fighting COVID-19, which could not only help in this pandemic but the next one too. In the near future, artificial Intelligence based on machine learning could be utilised to detect early stages of outbreaks, though privacy issues in training data for machine learning could cause problems.

\section{CONCLUSION}

Various ICT tools have been implemented and used to block misinformation, guide authentic information, support clinical decisions, and enable contact tracing, among other applications. Although ICT has become indispensable in the fight against COVID-19, there are still many suspicions regarding its effects. Further research and development should be performed for the next pandemic. 


\section{References}

1. Zarocostas J. How to fight an infodemic. Lancet. 2020;395:676.

2. The Sun. Why are people panic buying toilet paper, handwash, and pasta? Available at: https://www. thesun.co.uk/news/11130055/whyare-people-panic-buying-toiletpaper/. Last accessed: 17 November 2020.

3. Chang A et al. Cleaning and disinfectant chemical exposures and temporal associations with COVID-19 - National Poison Data System, United States, January 1, 2020-March 31, 2020. MMWR Morb Mortal Wkly Rep. 2020;69:496-8.

4. The International Telecommunication Union (ITU). No scientific basis between 5G and COVID-19. Available at: https://www.itu.int/en/Pages/ COVID-19/5g-covid-19-statement. aspx. Last accessed: 17 November 2020
5. The Centers for Disease Control and Prevention (CDC). Reducing stigma. Available at: https://www.cdc.gov/ coronavirus/2019-ncov/daily-lifecoping/reducing-stigma.html. Last accessed: 17 November 2020.

6. Lin $\mathrm{C}$ et al. Policy decisions and use of information technology to fight COVID-19, Taiwan. Emerging Infect Dis. 2020:26:1506-12.

7. John Hopkins University \& Medicine. Coronavirus resource center. 2020. Available at: https://coronavirus.jhu. edu/. Last accessed: 18 November 2020.

8. World Health Organization (WHO). WHO Coronavirus Disease (COVID-19) Dashboard. 2020. Available at: https://covid19.who.int/. Last accessed: 18 November 2020

9. Tokyo Metropolitan Government. Updates on COVID-19 in Tokyo. 2020.
Available at: https://stopcovid19. metro.tokyo.lg.jp/en. Last accessed: 18 November 2020.

10. Wang $S$ et al. A deep learning algorithm using CT images to screen for corona virus disease (COVID-19). medRxiv. 2020:DOl:10.1101/2020.02.14 .20023028

11. Li M et al. Development of an openEHR Template for COVID-19 based on clinical guidelines. J Med Internet Res. 2020;22:e20239.

12. Ferretti $L$ et al. Quantifying SARSCoV-2 transmission suggests epidemic control with digital contact tracing. Science. 2020;368(6491).

13. World Health Organization (WHO). Guidance for managing ethical issues in infectious disease outbreaks. 2016. Available at: https://apps.who.int/iris/ handle/10665/250580. Last accessed: 17 November 2020. 